\title{
Distribution System Planning With Battery Storage Using Multiperiod Optimal Power Flow
}

\author{
$1^{\text {st }}$ Jaap Pedersen \\ Reiner Lemoine Institute \\ Berlin, Germany \\ jaap.pedersen@rl-institut.de
}

\author{
$2^{\text {nd }}$ Birgit Schachler \\ Reiner Lemoine Institute \\ Berlin, Germany \\ birgit.schachler@rl-institut.de
}

\author{
$3^{\text {rd }}$ Anya Heider \\ Reiner Lemoine Institute \\ Berlin, Germany \\ anya.heider@rl-institut.de
}

\author{
$4^{\text {th }}$ Guido Pleßmann \\ Reiner Lemoine Institute \\ Berlin, Germany \\ guido.plessmann@rl-institut.de
}

\begin{abstract}
The ongoing energy transition introduces new challenges for distribution networks and brings about the need to expand existing power grid capacities. In order to contain network expansion and with it economic costs, utilization of various flexibility o ptions to r educe expansion n eeds is discussed. This paper proposes a multiperiod optimal power flow (MPOPF) approach with a new continuous network expansion formulation to optimize the deployment of flexibility o $p$ tions $u n$ der the objective of minimizing network expansion costs.

In a comparison of the newly proposed continuous network expansion formulation with an existing mixed integer formulation and a continuous interpretation of the latter the here proposed formulation is shown to be useful in order to obtain a solvable problem and contain computational efforts.

The presented MPOPF including the flexibility options storage units and curtailment is then assessed on synthetic medium voltage grids and applied to evaluate the benefit of a combined vs. a stepwise optimization of these flexibility o $p$ tions. I $t$ is demonstrated that using a local solver the proposed approach is applicable and yields a solution in reasonable time. Furthermore, it is shown that the combined optimization generally leads to a more efficient $u t$ ilization of $t h$ e c o nsidered fl ex ibility options and therefore lower grid expansion costs than the stepwise consideration.
\end{abstract}

Keywords-multiperiod optimal power flow, distribution network planning, battery storage, open source

\section{INTRODUCTION}

The trend towards a $100 \%$ renewable energy production comes along with a growing share of intermittent wind and solar power plants as well as increasing electrification of the heat and transport sectors. These transitions introduce new challenges for network operators and bring about the need to expand existing power grid capacities [1]. For society it is of interest to find e conomically ef ficient so lu tions. To this end, numerous studies examine measures to reduce network expansion needs, such as storage units, demand response, curtailment, reactive power management, etc. These measures are in this paper referred to as flexibility options.

Most distribution grid studies, e.g. [1]-[3], assess the benefit of individual flexibility options to m itigate $\mathrm{n}$ etwork expansion needs through variant calculations. In [4] a sequential introduction of the flexibility o ptions $\mathrm{c}$ urtailment a nd s torage units using heuristics is examined. Other studies use optimization approaches to minimize network expansion costs [5], [6]. However, these studies consider the transmission grid and use linearized optimal power flow (OPF) a p proaches that fail when it comes to low and medium voltage distribution grids [7]. [8] presents an iterative algorithm making the linearized MPOPF applicable for optimal sizing and siting of storage units in a low voltage network. In [9] a nonlinear MPOPF is presented to optimize storage operation. Both studies however do not consider network expansion as well as further flexibility options.

The aim of this paper is to propose a MPOPF to minimize network expansion costs considering optimal placement and operation of storage units and further flexibility options simultaneously. In general, the OPF determines an optimal operating point for an electric power system with respect to a specific objective, typically minimization of generation costs or grid losses, such that the technical and physical constraints, e.g. voltage bounds and power flow equations, respectively, are not violated [10]. While network expansion may be assessed for single worst case operating points, particularly the state-ofcharge dynamics of storage units introduce temporal coupling, wherefore the problem needs to be extended to an MPOPF formulation.

Due to the power flow equations, the OPF problem is a non-convex, nonlinear, large-scale optimization problem and, hence, hard to solve [11]. Network planning is usually approached by solving a mixed integer programming (MIP) problem [12]. As a MIP approach introduces additional complexity, this work proposes a continuous representation of the network expansion planning (NEP) by introducing continuous variables for technical parameters of the lines, i.e. maximal allowed current, resistance and reactance.

The paper is organized as follows. Section II presents the problem formulation including the derivation of the continuous formulation of network expansion variables used in this paper. Section III-A evaluates the continuous NEP against the common MIP approach. In Section III-B, the proposed MPOPF considering the usage of storage units and possible curtailment to avoid network expansion needs is assessed. The paper is concluded in Section IV. Throughout this work a fully open-source and open-data approach is pursued. The presented formulations are implemented in the open-source tool eDisGo [13]; the input data used is available online [14]-[16]. 


\section{PROBLEM FORMULATION}

This section presents the MPOPF formulation with network expansion and storage units used in this paper. An overview of the used nomenclature is given in Table I.

TABLE I: Nomenclature

\begin{tabular}{|c|c|}
\hline Symbols & Definition \\
\hline$G$ & graph $G=(N, E)$ representing a network \\
\hline$N$ & set of nodes in a network, $N=\{0,1, \ldots, n\}$ \\
\hline$E$ & set of edges in a network \\
\hline & set of nodes with storage units in a network \\
\hline$(i, j), i j$ & line from node $i$ to node $j$ \\
\hline & imaginary unit \\
\hline$A^{*}$ & complex conjugate of a complex variable $\mathrm{A}$ \\
\hline$\overline{\mathbf{y}}$ & upper bound of a variable $y$ \\
\hline $\mathbf{y}$ & lower bound of a variable $y$ \\
\hline $\bar{S}_{i j}$ & apparent power flow over line $i j$, \\
\hline$P_{i j}, Q_{i j}$ & active and reactive power flow over line $i j$ \\
\hline$I_{i j}$ & complex current over line $i j$ \\
\hline$\ell_{i j}$ & squared current magnitude over line $i j$ \\
\hline$z_{i j}$ & impedance of line $i j, z_{i j}=r_{i j}+\boldsymbol{j} x_{i j}$ \\
\hline$r_{i j}, x_{i j}$ & resistance and reactance of line $i j$ \\
\hline & voltage magnitude at bus $i$ \\
\hline$v_{i}$ & squared voltage magnitude at bus $i$ \\
\hline$s_{i}$ & apparent power injection at bus $i, s_{i}=p_{i}+j q_{i}$ \\
\hline$p_{i}, q_{i}$ & $\begin{array}{l}\text { active and reactive power injection at bus } i, \\
p_{i}:=p^{g}+p^{c} \quad q_{i}:=q^{g}+q^{c}\end{array}$ \\
\hline$p_{i}^{g}, q_{i}^{g}$ & $\begin{array}{l}\text { active and reactive power output of generator at } \\
\text { bus } i\end{array}$ \\
\hline$p_{i}^{c}, q_{i}^{c}$ & active and reactive power consumption at bus $i$ \\
\hline & time horizon with $t$ timesteps of length $\mathbf{T}_{\mathbf{S}}$ \\
\hline & length of time step \\
\hline$u_{c, i}, u_{d, i}$ & charging and discharging rate of storage at bus $i$ \\
\hline$\eta_{c, i}, \eta_{d, i}$ & $\begin{array}{l}\text { charging and discharging efficiency of storage at } \\
\text { bus } i\end{array}$ \\
\hline$e_{i}^{t}, e_{i}^{t+1}$ & $\begin{array}{l}\text { state of charge of storage at bus } i \text { at time } t \text { and } \\
\text { time } t+1\end{array}$ \\
\hline & initial impedance of line $i j, \mathbf{z}_{i j}^{0}=\mathbf{r}_{i j}^{0}+j \mathbf{x}_{i j}^{0}$ \\
\hline $\mathbf{r}_{i j}^{0}, \mathbf{x}_{i j}^{0}$ & initial resistance and reactance of line $i j$ \\
\hline$I_{i j}^{\max }$ & maximal allowed current flow over line $i j$ \\
\hline $\mathbf{I}_{i j}^{\max , 0}$ & initial maximal allowed current flow over line $i j$ \\
\hline$n_{i j}$ & number of parallel lines between node $i$ and node $j$ \\
\hline$c_{i j}$ & costs of line $i j$ \\
\hline
\end{tabular}

\section{A. Branch flow model}

Key constraints of the OPF are the power flow equations represented here by the non-convex, nonlinear branch flow model (BFM) that was first introduced by Baran and $\mathrm{Wu}$ [17], [18]. The BFM focuses on currents and powers on the branches, in contrast to the more popular bus injection model (BIM) that focuses on the nodal variables such as voltages, currents and power injections. Both formulations, however, are equivalent as shown in [19].

This study considers medium-voltage (MV) distribution networks. Under normal conditions MV networks are commonly operated as isolated half-rings [20], resulting in a tree topology. They can therefore be treated as radial networks. Let $G=(N, E)$ be a connected directed tree representing a radial MV network where each node in $N$ represents a bus and each edge in $E$ represents a distribution line. The nodes are indexed by $i=0,1, \ldots, n$. Denote an edge by $(i, j)$ if it connects node $i$ with node $j$.

For each edge $(i, j) \in E$, let $I_{i j}$ be the complex current and $S_{i j}=P_{i j}+\mathbf{j} Q_{i j}$ be the sending-end complex power flow from bus $i$ to bus $j$, with $P_{i j}$ and $Q_{i j}$ denoting the active and reactive power flow, respectively. Additionally, let $z_{i j}=$ $r_{i j}+\boldsymbol{j} x_{i j}$ be the complex impedance on the line between bus $i$ and bus $j$ with resistance $r$ and reactance $x$. For each node $i \in N$, let $V_{i}$ be the complex voltage and $s_{i}=p_{i}+j q_{i}$ be the complex power injection at bus $i$, with $p_{i}$ and $q_{i}$ denoting the active and reactive power injection, respectively. The active and reactive power injection consist of power generation and consumption $p_{i}:=p_{i}^{g}-p_{i}^{c}$ and $q_{i}:=q_{i}^{g}-q_{i}^{c}$.

Consider a radial network with orientation towards the substation bus. For each node $i$ and each edge $(i, j)$, the variables $(S, V, I)$ have to satisfy

Ohm's law

$$
V_{i}-V_{j}=z_{i j} I_{i j} \quad \forall(i, j) \in E,
$$

the definition of the branch power flow

$$
S_{i j}=V_{i} I_{i j}^{*} \quad \forall(i, j) \in E,
$$

and the power balance at each bus

$$
S_{j k}-\sum_{i: i \rightarrow j}\left(S_{i j}-z_{i j}\left|I_{i j}\right|^{2}\right)=s_{j} \quad \forall j \in N .
$$

As customary, it is assumed that the complex voltage at the substation bus is given.

Substituting (2) into (1) yields:

$$
V_{j}=V_{i}-z_{i j} \frac{S_{i j}^{*}}{V_{i}^{*}} \quad \forall(i, j) \in E
$$

Taking the magnitude squared of (2) and (4) and writing the equations in terms of real variables, one obtains the BFM [21]:

$\begin{array}{ll}p_{j}=P_{j k}-\sum_{i: i \rightarrow j}\left(P_{i j}-r_{i j} \ell_{i j}\right) & \forall j \in N \\ q_{j}=Q_{j k}-\sum_{i: i \rightarrow j}\left(Q_{i j}-x_{i j} \ell_{i j}\right) & \forall j \in N\end{array}$

$v_{j}=v_{i}-2\left(r_{i j} P_{i j}+x_{i j} Q_{i j}\right)+\left(r_{i j}^{2}+x_{i j}^{2}\right) \ell_{i j} \quad \forall(i, j) \in E$

$v_{i} \ell_{i j}=P_{i j}^{2}+Q_{i j}^{2}$

$\forall(i, j) \in E$

where $l_{i j}=\left|I_{i j}\right|^{2}$ denotes the squared current magnitude and $v_{i}=\left|V_{i}\right|^{2}$ the squared voltage magnitude.

In the literature it is often called the relaxed branch flow model, as information of the voltage and current angles, $\angle V_{i}$ and $\angle I_{i j}$, respectively, are lost, and the set of variables $(P, Q, v, \ell)$ is a subset of the complex variables $(S, V, I)$, see 
e.g. [21], [22], [23]. However, in [21] it is proven that for radial networks, i.e. $G$ is a tree, one can easily recover the angles from the solution of the relaxed BFM. Therefore, the term BFM is used for the power flow equations (5) - (8) throughout this paper. Note that the time dependence of the equations is omitted for brevity.

\section{B. Network expansion}

Distribution grid operators are obliged to guarantee a safe and reliable electricity supply at a certain quality. To ensure fulfillment of these objectives, thermal loading of network equipment and voltage deviations must be kept within certain limits. These limits are reflected by the following constraints:

$$
\begin{array}{ll}
\ell_{i j} \leq\left|I_{i j}^{\max }\right|^{2} & \forall(i, j) \in E \\
\underline{\boldsymbol{v}_{\boldsymbol{i}}} \leq v_{i} \leq \overline{\boldsymbol{v}_{\boldsymbol{i}}} & \forall i \in N
\end{array}
$$

where $I_{i j}^{\max }$ is the current limit of the line between bus $i$ and bus $j$ and $\boldsymbol{v}_{\boldsymbol{i}}$ and $\overline{\boldsymbol{v}_{\boldsymbol{i}}}$ are the lower and upper bound of the voltage at node $i$, respectively.

When these constraints cannot be met, network expansion is required. Network expansion is usually approached by solving a MIP problem, where $n$ identical parallel lines may be installed between any two buses, as described for example in [11] and [12]. In that case, line impedances are technical parameters and fixed for each line. Again, each node $i$ and each line $(i, j)$ in $E$ has to satisfy the power flow equations presented in the previous section II-A, resulting in the following constraints, written in terms of real variables:

$$
\begin{array}{lr}
p_{j}=\sum_{k: j \rightarrow k} n_{j k} P_{j k}-\sum_{i: i \rightarrow j} n_{i j}\left(P_{i j}-r_{i j} \ell_{i j}\right) & \forall j \in N \\
q_{j}=\sum_{k: j \rightarrow k} n_{j k} Q_{j k}-\sum_{i: i \rightarrow j} n_{i j}\left(Q_{i j}-x_{i j} \ell_{i j}\right) & \forall j \in N \\
v_{j}=v_{i}-n_{i j}\left(2\left(r_{i j} P_{i j}+x_{i j} Q_{i j}\right)+\right. & (12) \\
\left.\left(r_{i j}^{2}+x_{i j}^{2}\right) \ell_{i j}\right) & \forall(i, j) \in E \\
& \forall(i, j) \in E \\
v_{i} \ell_{i j}=P_{i j}^{2}+Q_{i j}^{2} & (13) \\
0 \leq \ell_{i j} \leq \overline{\boldsymbol{\ell}}_{\boldsymbol{i j}} & \forall(i, j) \in E \\
\underline{\boldsymbol{v}}_{\boldsymbol{i}} \leq v_{i} \leq \overline{\boldsymbol{v}}_{\boldsymbol{i}} & \\
1 \leq n_{i j} \leq \boldsymbol{n}_{\boldsymbol{m a x}}, n_{i j} \in \mathbb{Z} & \forall i \in N(15) \\
& \forall(i, j) \in E
\end{array}
$$

As only whole numbers of lines can be installed, the constraints (11)-(13) containing the integer variable $n$ become mixed integer nonlinear programming (MINLP) constraints. As MINLP problems are generally harder to solve than nonlinear programming (NLP) problems, this motivates the reformulation of the network expansion problem into a continuous NLP problem shown in the following.
The reformulation utilizes the characteristics of resistances and inductor units in parallel circuits [24]. The total resistance $r$ of a parallel circuit with $N$ equal resistance units $r_{0}$ and the total inductance $L$ for $N$ equal non-coupled, i.e. noninteracting magnetic fields, inductor units $L_{0}$ can be calculated as:

$$
\begin{aligned}
& r=\frac{r_{0}}{N} \\
& L=\frac{L_{0}}{N}
\end{aligned}
$$

The reactance of a circuit $x$ is proportional to its inductance and the frequency of the current in the electrical power grid, wherefore (19) can be written as:

$$
x=\frac{x_{0}}{N}
$$

Denote the maximal allowed current over a single circuit as $I^{\max , 0}$ and consider $N$ equal parallel circuits. The total maximal allowed current, denoted as $I^{\max }$, can then be written as:

$$
I^{\max }=N I^{\max , 0} \Leftrightarrow \quad \Leftrightarrow=\frac{I^{\max }}{I^{\max , 0}}
$$

Plugging (21) into (18) and (20) the resistance and reactance of each line $(i, j) \in E$ become:

$$
\begin{array}{rlrl}
r_{i j} I_{i j}^{\max } & =r_{i j}^{0} I_{i j}^{\max , 0} & & \forall(i, j) \in E \\
x_{i j} I_{i j}^{\max }=x_{i j}^{0} I_{i j}^{\max , 0} & & \forall(i, j) \in E
\end{array}
$$

For lines that can be expanded, $r_{i j}$ and $x_{i j}$ become variables so that the power flow equations (5) - (8) and constraints introduced in (9) and (16) have to be satisfied with the set of variables $\left(P, Q, v, \ell, I^{\max }, r, x\right)$. Letting the ratio of $I^{\max }$ and $I^{\max , 0}$ be continuous, the MINLP turns into an NLP.

\section{Storage units}

Storage units can serve as a flexibility to the power network by shifting variable renewable generation from high feedin, off-peak load periods to low feed-in, high load periods and therewith reduce line loadings and voltage deviations. However, in order to do so it must be ensured that the state of charge (SOC) of the storage units allows for the required energy intake or discharge, respectively. Therefore, to assess storage potential to reduce grid load, a broader period of time needs to be analyzed, requiring a MPOPF with temporal coupling, due to the SOC evolution.

[25] gives an overview of how storage units are handled in MPOPF problems. Here, the formulation from [9] is used. The set of nodes with storage units is denoted as $S$. Let $\mathcal{T}=$ $\{0, \ldots, T\}$ be the time horizon, in which the time step of length $T_{s}$ is denoted as $t$, and $e^{t}$ be the SOC at time step $t$. The SOC dynamic is formulated as:

$$
\boldsymbol{T}_{\boldsymbol{s}}\left(\boldsymbol{\eta}_{\boldsymbol{c}, \boldsymbol{i}} u_{c, i}^{t}-\frac{u_{d, i}^{t}}{\boldsymbol{\eta}_{\boldsymbol{d}, \boldsymbol{i}}}\right)=e_{i}^{t+1}-e_{i}^{t} \quad \forall i \in S, \forall t \in \mathcal{T}
$$


where $\eta_{c}$ and $\eta_{d}$ denote the charging and discharging efficiency, and $u_{c}$ and $u_{d}$ the charging and discharging rate. Furthermore, the storage units are subject to upper and lower limits for charging and discharging rate as well as the SOC:

$$
0 \leq u_{c, i} \leq \overline{\boldsymbol{u}_{\boldsymbol{c}, \boldsymbol{i}}}, \quad 0 \leq u_{d, i} \leq \overline{\boldsymbol{u}_{\boldsymbol{d}, \boldsymbol{i}}}, \quad 0 \leq e_{i} \leq \overline{e_{i}} \quad \forall i \in S
$$

To prevent storage units of simultaneous charging and discharging the following complementary constraint is introduced:

$$
u_{c, i} u_{d, i}=0 \quad \forall i \in S
$$

This non-convex complementary constraint of the charging/discharging rate is in [9] relaxed by introducing the convex hull of the constraint, as given by equation (27).

$$
u_{c, i} u_{d, i}=0 \quad \Rightarrow \quad u_{c, i} \leq-\left(\frac{\overline{u_{c, i}}}{\overline{u_{d, i}}}\right) u_{d, i}+\overline{u_{c, i}} \quad \forall i \in S
$$

Furthermore, we require a periodic end-value of the SOC, with $e^{0}$ being the initial state of charge and $e^{T+1}$ being the final state of charge:

$$
e_{i}^{0}=e_{i}^{T+1} \quad \forall i \in S
$$

\section{RESULTS AND DISCUSSION}

This section first presents the results of the evaluation of different network expansion formulations conducted on small IEEE test cases. Insights from the preliminary analysis are utilized in the next evaluation step in which flexibility options are added to the network expansion problem and evaluation is extended to larger synthetic MV grids, in order to assess feasibility and applicability of the proposed MPOPF approach to larger problems.

\section{A. Evaluation of network expansion formulations}

The analysis presented here serves as an evaluation of the in section II-B proposed continuous network expansion formulation, referred to as $N L P$ formulation. The $N L P$ formulation is compared to the discrete network expansion formulation, as well introduced in section II-B and further referred to as MIP formulation, and a continuous interpretation of the network expansion variables in the discrete formulation, referred to as $c M I P$. The formulations are evaluated with respect to computing time and exactness. The objectives and constraints of the three formulations are as follows:

$$
\text { MIP: } \min _{\substack{p_{g}^{t}, q_{g}^{t}, v^{t}, \ell^{t}, P^{t}, Q^{t}, n}} \sum_{(i, j) \in E} c_{i j} n_{i j}+\sum_{t \in \mathcal{T}} \sum_{i \in N} C_{g}\left(p_{g, i}^{t}\right)
$$

$$
\text { s.t. }(\forall t \in \mathcal{T})
$$

$$
\text { (11) }-(17)
$$$$
\underline{\boldsymbol{p}_{\boldsymbol{i}}} \leq p_{i} \leq \overline{\boldsymbol{p}_{\boldsymbol{i}}}, \quad \underline{\boldsymbol{q}_{\boldsymbol{i}}} \leq q_{i} \leq \overline{\boldsymbol{q}_{\boldsymbol{i}}} \quad \forall i \in N
$$

cMIP: $\min (29)$

$$
\text { s.t. }(\forall t \in \mathcal{T})
$$

$$
\begin{aligned}
& (11)-(16) \\
& 1 \leq n_{i j} \leq \boldsymbol{n}_{\text {max }}, \quad n_{i j} \in \mathbb{R} \quad \forall(i, j) \in E
\end{aligned}
$$

$$
\begin{aligned}
& \text { NLP: } \min _{\begin{array}{c}
\min _{g}^{t}, q_{g}^{t}, v^{t}, \\
\ell^{t}, P^{t}, Q^{t}, I^{\max }, r, x
\end{array}}\left(\sum_{(i, j) \in E} c_{i j} I_{i j}^{\max }+\right. \\
&\left.\sum_{t \in \mathcal{T}} \sum_{i \in N} C_{g}\left(p_{g, i}^{t}\right)\right)
\end{aligned}
$$

s.t. $(\forall t \in \mathcal{T})$

$$
\text { (5) - (10), (22), (23), (30) }
$$

Here, $C_{g}\left(p_{g, i}^{t}\right)$ is a quadratic cost function with cost factors specified in the corresponding NESTA case file.

These non-convex problems are solved using the noncommercial global solver for mixed integer nonlinear programming SCIP v.6.0.1 [26], which is based on a combined approach of Branch-and-bound and LP relaxations [27]. By empirical observation, the implemented presolving was disadvantageous and turned off. All simulations are performed on an HP ProBook with Intel Core i5-4210M (2.60GHz, 4 threads) and $8 \mathrm{~GB}$ memory. For all of the instances, a time limit of $1000 \mathrm{~s}$ and a primal-dual gap limit of $1 \%$ is chosen. The primal-dual gap is defined as $g a p \%=\frac{\text { primal-dual }}{\text { primal }} \times 100$.

The study is conducted using two radial IEEE networks used in [28] and provided through the NESTA library, a collection of established power network test cases [16]. The grids used here are the 30 and 57 bus cases nesta_case30_kds_rad and $n e s t a \_c a s e 57 \_k d s \_r a d$, respectively. For the purpose of this analysis slight changes of the test cases are made. For once, the active power output of generators at the buses 11 and 13 in case of the 30 bus case, and 2, 6, and 9 in case of the 57 bus case are fixed to simulate non-dispatchable generation, e.g. weather-dependent renewable energy sources. Furthermore, in order to represent the increase in distributed generation and their volatility, the nominal power of the non-dispatchable generators is doubled compared to the original NESTA cases and a random feed-in time series with values between 0 and 1 is introduced by which the power injection $p_{g, i}^{t}$ for all generators $i$ in the set of the non-dispatchable generators is multiplied. The demand is fixed and the feed-in of dispatchable 
generators is optimized. Key figures of the test cases are summarized in table II. The optimization is conducted for time horizons of $\mathcal{T}=1 \ldots 8$.

TABLE II: Key figures of IEEE test cases

\begin{tabular}{|l|l|c|c|}
\hline \multicolumn{2}{|l|}{} & nesta_case30 & nesta_case57 \\
\hline Number of buses & $|N|$ & 30 & 57 \\
\hline \multirow{2}{*}{ Load } & $P_{\max }^{d}$ & $1.42 \mathrm{MW}$ & $4.7 \mathrm{MW}$ \\
& $Q_{\max }^{d}$ & $0.63 \mathrm{MVar}$ & $1.11 \mathrm{MVar}$ \\
\hline \multirow{2}{*}{ Dispatchable } & $P_{\max }^{g}$ & $3.40 \mathrm{MW}$ & $11 \mathrm{MW}$ \\
generation & $Q_{\max }^{g}$ & $1.30 \mathrm{MVar}$ & $4.15 \mathrm{MVar}$ \\
& $Q_{\min }^{g}$ & $-0.6 \mathrm{MVar}$ & $-3.30 \mathrm{MVar}$ \\
\hline Non- & $P_{\max }^{g}$ & $4.00 \mathrm{MW}$ & $6.00 \mathrm{MW}$ \\
dispatchable & $Q_{\max }^{g}$ & $0.96 \mathrm{MVar}$ & $0.84 \mathrm{MVar}$ \\
generation & $Q_{\min }^{g}$ & $0.00 \mathrm{MVar}$ & $-0.58 \mathrm{MVar}$ \\
\hline
\end{tabular}

Fig. 1 gives the computing time (left) of the instances and their primal-dual gap (right) for global optimality over the considered time spans for the two test cases. The figure shows that in case of the MIP formulation, a primal solution for the 30 bus case can only be found within the given time limit for a time horizon of $\mathcal{T}=1$, with a primal-dual gap of $24 \%$. For the 57 bus case, SCIP manages to find solutions for up to five time steps with primal-dual gaps of generally around $15 \%$.
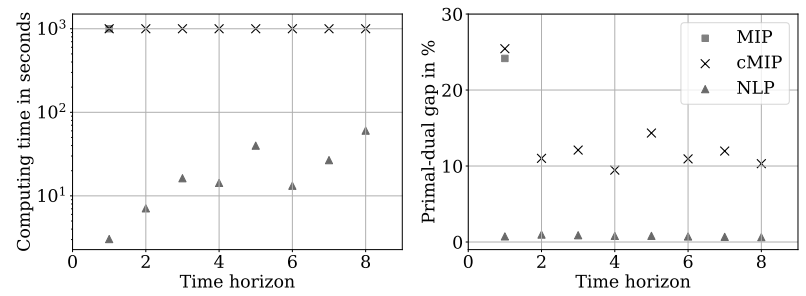

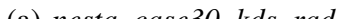
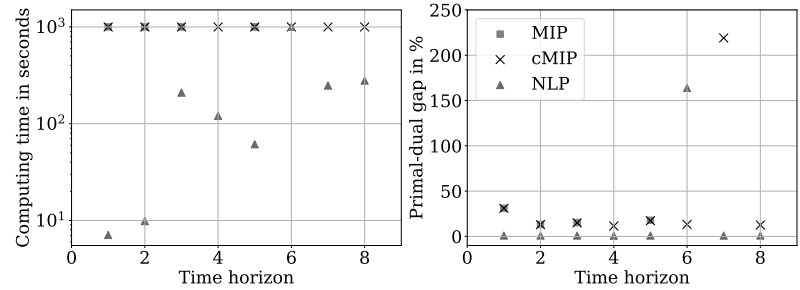

(b) nesta_case57_kds_rad

Fig. 1: Computing time and primal-dual gap for solving the $M I P$ (square) and the two continuous nonlinear problem formulations $c M I P$ (cross) and NLP (triangle) for the 30 (top) and 57 (bottom) bus network to global optimality within specified time and gap limits using SCIP.

In contrast to the $M I P$ formulation, primal solutions for the two continuous network expansion formulations, $c M I P$ and $N L P$, are found for all considered time spans. The final objective values of the formulations are similar for each time horizon. However, whereas in case of the NLP formulation the primal-dual gap of the solution can be closed to up to at least $1 \%$ in less than $300 \mathrm{~s}$ for all but one instance, the primal-dual gap in case of the $c M I P$ formulation can in most cases only be closed to values between $10 \%$ to $15 \%$ within the given time limit of $1000 \mathrm{~s}$. For all cases where a solution can be found in case of the MIP formulation, the primal-dual gap of the $c M I P$ and the $M I P$ formulation are similar.

In the case of the 57 bus network two strong deviations of the primal-dual gap can be observed. These deviations occur for the instances $c M I P: \mathcal{T}=7$ and $N L P: \mathcal{T}=5$ with gaps of $>200 \%$ and $>150 \%$, respectively. This is assumed to result from choices of primal heuristics in SCIP. The primal solution of most of the instances is found by the local interior point method, but in these mentioned cases the large neighborhood search heuristic is used.

The analysis shows that the MIP formulation does not yield a solution in reasonable time for the small problems considered here. As it is planned to apply the optimization to real grid data, which will in most cases be larger than the NESTA cases used here, and furthermore add flexibility options that will introduce a temporal coupling, such as storage units, making the problem even harder, the MIP formulation is not regarded applicable for the planned use cases.

As expected, the continuous formulations outperform the MIP formulation with respect to computation time, as mixed integer problems are, in general, harder to solve. However, network expansion costs are underestimated in a continuous approach, which needs to be considered when including other costs in the objective function and when interpreting results.

Compared to the $c M I P$ formulation, the $N L P$ formulation performs significantly better. In both test cases, the $N L P$ reduces computation time by at least an order of ten, in order to find a solution and prove global optimality with up to $1 \%$ and does therefore constitute a more suitable approach. A reason for the better performance could be that the NLP formulation has less quadratic terms than the $c M I P$ formulation due to the multiplication of equations (11) - (13) with the number of lines $n$ used in the $c M I P$ formulation.

\section{B. Continuous network expansion with multiple flexibility op- tions}

In this part feasibility and applicability of the proposed MPOPF with continuous network expansion for larger problems and multiple flexibility options is assessed.

The current study considers the two flexibility options storage units and curtailment. In the case of storage units the constraints introduced in section II-C apply. Furthermore, a maximum storage capacity $\boldsymbol{E}_{\text {storage }}$ that may be distributed in the medium voltage grid is given, introducing the following constraint:

$$
\sum_{\forall i \in S} \overline{e_{i}} \leq \boldsymbol{E}_{\text {storage }}
$$

In the optimization the optimal storage sizing, siting and operation under the given constraints are determined.

Concerning curtailment of wind and solar resources it is here assumed that given curtailment requirements $\boldsymbol{P}_{\text {curtail }}$ 
can be optimally allocated to all generation units within the grid. The curtailed power of generation units at node $i$ is calculated as the difference between the weather dependent availability of generators at that node $\boldsymbol{p}_{\boldsymbol{i}, \boldsymbol{g}}$ and the actual feedin $p_{i, g}$. Therewith, the additional constraints to incorporate curtailment can be formulated as:

$$
\begin{aligned}
& \sum_{\forall i \in E}\left(\boldsymbol{p}_{\boldsymbol{i}, \boldsymbol{g}}-p_{i, g}\right)=\boldsymbol{P}_{\text {curtail }} \\
& 0 \leq p_{i, g} \leq \boldsymbol{p}_{\boldsymbol{i}, \boldsymbol{g}} \quad \forall i \in N
\end{aligned}
$$

Two scenarios incorporating both flexibility options are evaluated. One is the All-flex-combined scenario, where both flexibility options are optimized in one optimization step. The other one is the All-flex-serial scenario, where first curtailment and in a second step storage units are optimized, in order to assess the potential advantage of a combined optimization. As benchmark to assess the flexibility options against, network expansion is optimized without flexibility options. The scenario is referred to as No-flex, with the same constraints as the $N L P$ formulation in section III-A.

$$
\begin{aligned}
& \text { No-flex: } \min _{\substack{p_{g}^{t}, q_{g}^{t}, v^{t}, \ell^{t}, P^{t}, Q^{t}, I^{\text {max }}, r, x}} \sum_{(i, j) \in E} C_{n e p}\left(I_{i j}^{\max }\right) \\
& \text { s.t. }(\forall t \in \mathcal{T})
\end{aligned}
$$

$$
\text { (5) - (10), (22), (23), (30) }
$$

The objective here is to minimize network expansion costs $C_{n e p}\left(I_{i j}^{\text {max }}\right)$. Network expansion costs consider cable costs $c_{i j}^{c a b l e}$ for additional capacity on each edge $(i, j) \in E$ :

$$
C_{n e p}\left(I_{i j}^{\max }\right)=\left(\frac{I_{i j}^{\max }}{I_{i j}^{\max , 0}}-1\right) c_{i j}^{\text {cable }}
$$

Earthwork costs are neglected as they either yield a MIP problem when considered only once for each expanded edge or introduce a negligible constant factor when added for each line or proportionally to the expanded capacity.

Besides the No-flex and two All-flex scenarios, scenarios to assess potential to reduce network expansion costs using only storage units or only curtailment are analysed, using the following formulations:

$$
\begin{aligned}
\text { Storage: } & \min (36) \\
\text { s.t. }(\forall t \in \mathcal{T}) & \\
& (5)-(10),(22)-(25),(27),(28),(30),(33)
\end{aligned}
$$

Curtailment: $\min (36)$

$$
\text { s.t. }(\forall t \in \mathcal{T})
$$

$$
\text { (5) - (10), (22), (23), (30), (34), (35) }
$$

In case of the All-flex-combined scenario constraints of the Storage and Curtailment scenarios are combined. As for the
All-flex-serial scenario first curtailment is optimized using the Curtailment formulation. Afterwards, storage units are optimized using the Storage formulation and fixing the generator feed-in to the optimized feed-in from the first optimization step.

The analyses are conducted on four synthetic MV grids, generated with the open-source software dingO [29]. dingO models MV grid topologies as open half-rings, taking into account local characteristics, such as demography and land use, as well as current planning principles. The grids chosen are MV grids 1618, 2761, 2776, 2799 with 140, 61, 108 and $167 \mathrm{MV}$ nodes, respectively. As the ding0 grids represent stable status quo networks without expansion needs, a scenario representing the future power system, called the eGo100 [5] scenario, is utilized. Time series of weather dependent generators and demand are as well taken from [5].

The curtailment time series are synthesized such that feedin in times of low demand is reduced by $15 \%$. The storage capacity $\boldsymbol{E}_{\text {storage }}$ to be distributed is for each grid set to $10 \mathrm{MW}$. Optimized storage units with a storage size of $<0.3 \mathrm{MW}$, which here signifies the minimum size at which units are connected to the MV grid, are discarded. To account for the discarded storage capacity in the evaluation of network expansion needs, network expansion needs considering only the nondiscarded storage units and their optimized operation from the storage optimization step are optimized in a subsequent optimization. It must further be noted that in order to reduce the complexity of the problem, storage units can only be build at nodes where grid problems occur. These critical nodes are identified prior to the storage optimization by a network expansion optimization.

The problems are optimized for time horizons of 8, 24 and 72 hours around the time step with the maximal negative residual load. For this study the local solver Interior Point Optimizer (Ipopt) [30] with the linear solver HSL_MA97 [31] is used. A local solver instead of a global solver is chosen to contain computational efforts, as this study serves as a first assessment of the proposed approach. However, for following more comprehensive studies usage of a global solver needs to be considered. All simulations are performed on a workstation with AMD Ryzen ThreadRipper 1950X (up to $4 \mathrm{GHz}, 64$ threads) and 64GB memory. While Ipopt is single-threaded, $H S L \_M A 97$ can utilize multiple cores. Here, HSL_MA97 is limited to three cores. For all of the instances, a tolerance of $1 \mathrm{e}-5$ and a maximum iteration number of 3,000 are chosen.

The analysis showed that for three of the sixty instances the solver failed to converge to a solution before reaching the maximum number of iterations. These are the Storage and Allflex-serial scenarios for grid 1618 and 72 time steps and the All-flex-combined scenario for grid 2799 and 72 time steps.

For all other instances an optimal solution was found.

Fig. 2 shows the mean computing time over all four grids for the different scenarios and number of time steps, as far as a solution was found. The plot shows the exponential increase in computing time with the number of time steps. However, even for the largest number of time steps considered here, 
computing time is kept at around five minutes. As expected, the No-flex scenario without flexibility options shows the smallest computing time. Interestingly enough, the combined optimization is for nearly all instances faster than the separate optimization of curtailment and storage units. It has to be noted though, that a local solver is used, wherefore the found solution does not necessarilv constitute the global optimum.

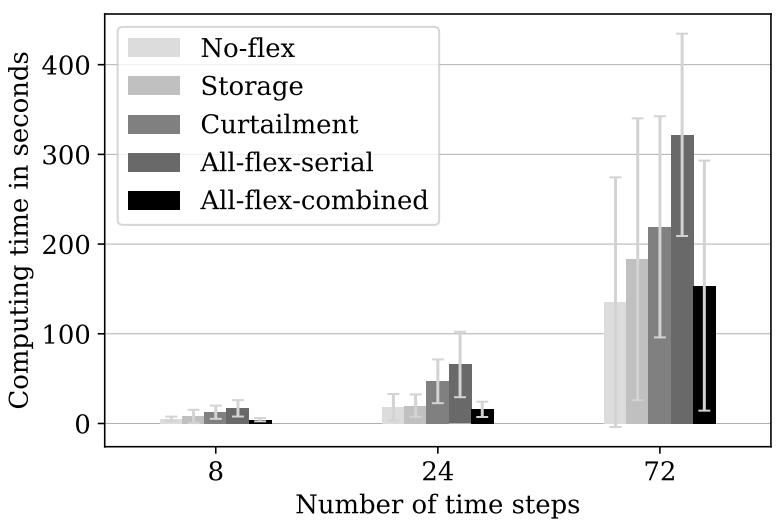

Fig. 2: Mean computing time to optimize the five scenario: for the three considered time horizons

The reduction of network expansion costs, i.e. the objectivt value, due to the deployment of flexibility options relative ts the benchmark scenario No-flex is shown in Fig. 3. If a solutior is found the flexibility option(s) reduced necessary networl expansion costs. Only in the case of the Curtailment scenaric for grid 2799 network expansion costs remained the sam for all considered time horizons. In most cases the combiner optimization of storage units and curtailment yielded slightly lower expansion costs compared to the separate optimization Exceptions to this occur for a time horizon of 8 time steps fo. all grids except grid 2761. In those cases the local solver dic not find the global optimum, otherwise cost reduction wouk at least be the same as for the separate optimization.

Fig. 4 shows the allocation of the two flexibility options for the combined (All-flex-combined) and separate (All-flexserial) optimization, exemplarily for grid 2761 and 72 time steps. As can be seen, the distribution of the curtailment requirement to the different nodes as well as storage sizing is slightly different between the two scenarios, leading to a more efficient deployment of the flexibilities and therewith less grid expansion needs in the combined optimization (cf. figure 3).

Two aspects have to be noted when assessing the reduction in network expansion costs due to flexibility options. Firstly, the cost reduction presented here comprises only the saved cable costs and does not, as stated before, include earthwork costs that in the MV are around four to seven times higher than cable costs and would further increase potential cost reduction. Secondly, due to the chosen approach of continuous network expansion, flexibility options may be deployed in such a way that the same discrete number of lines is required and therefore not lead to an actual reduction in grid expansion costs. This aspect needs to be analyzed more thoroughly in further studies.
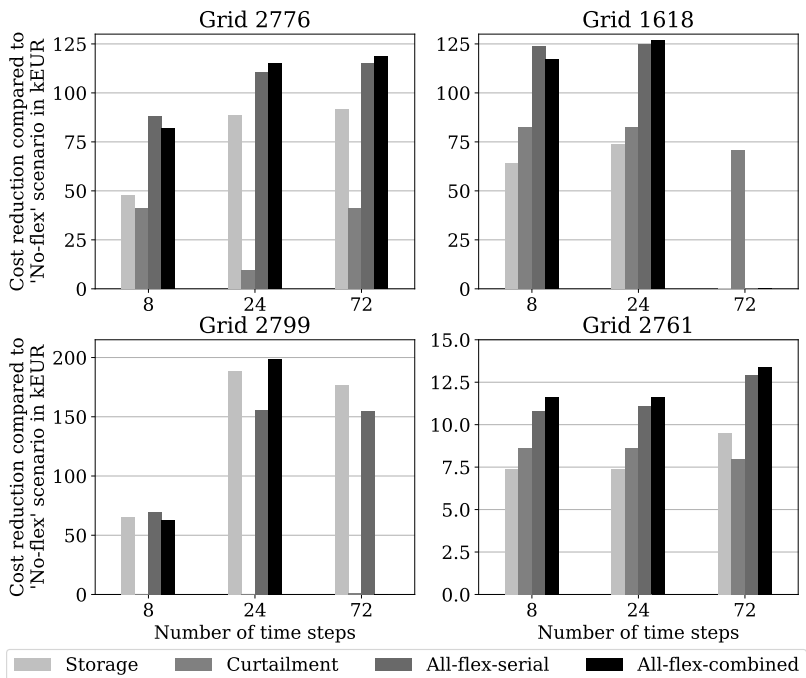

Fig. 3: Reduction of the objective value for the four scenarios with flexibility options relative to the benchmark scenario $\mathrm{No}$ flex

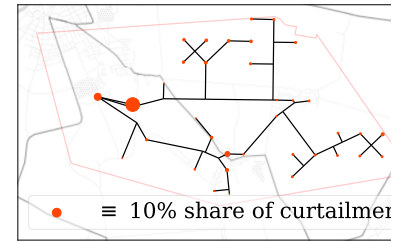

(a) Dilnoation of curtailmant

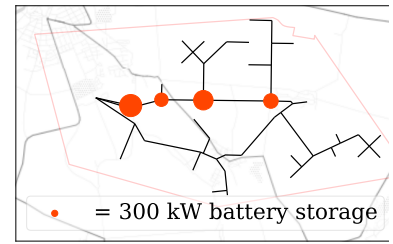
All-flex-serial scenario (c) Allocation of storage units

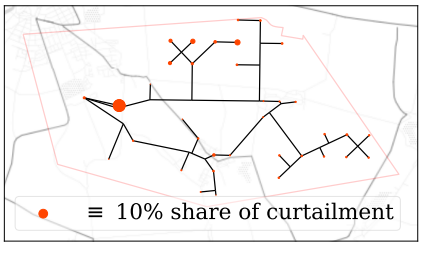

(h) silnoation of curtailmant in

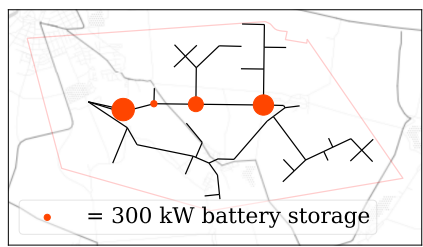

(d) Allocation of storage units in All-flex-combined scenario
Fig. 4: Comparison of curtailment and storage units allocation in All-flex-combined and All-flex-serial scenarios for grid 2761 and 72 time steps

\section{CONCLUSION}

In this paper an MPOPF approach to optimize network expansion needs considering the flexibility options storage units and curtailment has been proposed and assessed. The approach uses the branch flow formulation of the power flow equations and storage units formulation from [9]. Furthermore, it includes a new continuous network expansion formulation. In a preliminary analysis conducted on IEEE test cases the newly proposed network expansion formulation was compared to an existing discrete approach as well as a continuous interpretation of the discrete approach. The evaluation of the different network expansion approaches showed that the 
discrete approach only yields a solution in reasonable time for very small problems and is therefore not applicable. It was further shown that the proposed approach performs significantly better with respect to computation time than the continuous interpretation of the discrete formulation while yielding the same solution.

In a next step the proposed MPOPF including flexibility options was assessed using larger synthetic MV grids. It could be shown that the proposed approach is applicable and yields a solution in reasonable time. Furthermore, the flexibilities could be deployed more efficiently in the combined optimization of flexibility options, leading to lower network expansion costs compared to a serial optimization of storage units and curtailment. The benefit of the combined optimization is expected to become even more relevant with the growing number of flexibility options available and the associated growing complexity. However, an optimal solution or global optimum could not be found for all analyzed instances. Further research is therefore required to improve robustness and convergence of the optimization problem or to reduce its complexity.

One approach to reduce the complexity of the optimization problem that has become more and more popular in recent years is convex relaxations of the problem. Those relaxations enclose the non-convex feasible space, spanned by the power flow equations, and then use the fundamental property of convex optimization problems to solve the problem, i.e. any locally optimal point is also globally optimal [32]. Another approach is to reduce the number of variables of the optimization problem by temporal or spatial complexity reduction allowing the usage of global solvers with reasonable computing times. Both approaches are subject to ongoing research.

\section{REFERENCES}

[1] A. C. Agricola, B. Höflich, P. Richard, J. Völker, C. Rehtanz, G. M., B. Gwisdorf, J. Kays, T. Noll, J. Schwippe, A. Seack, J. Teuwsen, G. Brunekreeft, R. Meyer, and V. Liebert, "Ausbau- und Innovationsbedarf der Stromverteilnetze in Deutschland bis 2030 (kurz: dena-Verteilnetzstudie)," Endbericht, Deutsche Energie-Agentur GmbH (dena), 2012.

[2] J. Büchner, J. Katzfey, O. D. Floercken, A. Moser, H. Schuster, S. Dierkes, T. van Leeuwen, L. Verheggen, M. Uslar, and M. van Amelsvoort, " Moderne Verteilernetze für Deutschland (Verteilernetzstudie)," 2014.

[3] C. Rehtanz, M. Greve, U. Häger, Z. Hagemann, S. Kippelt, C. Kittl, M.-L. Kloubert, O. Pohl, F. Rewald, and C. Wagner, "Verteilnetzstudie für das Land Baden-Württemberg," tech. rep., ef.Ruhr GmbH, 2017.

[4] U. P. Müller, B. Schachler, M. Scharf, W.-D. Bunke, S. Günther, J. Bartels, and G. Pleßmann, "Integrated techno-economic power system planning of transmission and distribution grids," Energies, vol. 12, no. $11,2019$.

[5] U. P. Müller, B. Schachler, W.-D. Bunke, J. Bartels, M. Glauer, C. Büttner, S. Günther, E. Kötter, I. Cußmann, L. Hülk, M. Scharf, T. Mossakowski, and J. Wendiggensen, "Netzebenenübergreifendes Planungsinstrument - zur Bestimmung des optimalen Netz- und Speicherausbaus in Deutschland - integriert in einer OpenEnergy-Plattform," Projektabschlussbericht, 2019. https://openegoproject.wordpress.com/.

[6] L. H. Macedo, C. V. Montes, J. F. Franco, M. J. Rider, and R. Romero, "Milp branch flow model for concurrent ac multistage transmission expansion and reactive power planning with security constraints," IET Generation, Transmission Distribution, vol. 10, no. 12, pp. 3023-3032, 2016.

[7] T. Brown, J. Hörsch, and D. Schlachtberger, "PyPSA: Python for Power System Analysis," Journal of Open Research Software, vol. 6, no. 4, 2018.
[8] P. Fortenbacher, M. Zellner, and G. Andersson, "Optimal sizing and placement of distributed storage in low voltage networks," 062016.

[9] J. F. Marley, D. K. Molzahn, and I. A. Hiskens, "Solving multiperiod OPF problems using an AC-QP algorithm initialized with an SOCP relaxation," IEEE Transactions on Power Systems, vol. 32, pp. 35383548, Sep. 2017.

[10] D. K. Molzahn and I. A. Hiskens, "A Survey of Relaxations and Approximations of the Power Flow Equations," vol. 4, pp. 1-221, 01 2019.

[11] J. A. Taylor, Convex Optimization of Power Systems. Cambridge University Press, 2015.

[12] R. A. Jabr, "Optimization of AC Transmission System Planning," IEEE Transactions on Power Systems, vol. 28, pp. 2779-2787, Aug 2013.

[13] B. Schachler, A. Heider, J. Pedersen, G. Pleßmann, J. Amme, and B. Narasimhan, "eDisGo - electricity distribution grid optimization." https://github.com/openego/eDisGo, 2020.

[14] J. Amme and G. Pleßmann, "ding0 - distribution network generator." https://github.com/openego/ding0, 2020.

[15] IKS OvGU Mageburg and DLRVE Oldenburg and ZNES Flensburg and RLI Berlin, "OpenEnergy-platform (OEP)." http://openenergyplatform.org, 2018.

[16] C. Coffrin, D. Gordon, and P. Scott, "Nesta, the NICTA energy system test case archive," CoRR, vol. abs/1411.0359, 2014.

[17] M. E. Baran and F. F. Wu, "Optimal capacitor placement on radial distribution systems," IEEE Transactions on Power Delivery, vol. 4 pp. 725-734, Jan 1989.

[18] M. E. Baran and F. F. Wu, "Optimal sizing of capacitors placed on a radial distribution system," IEEE Transactions on Power Delivery, vol. 4 , pp. 735-743, Jan 1989 .

[19] B. Subhonmesh, S. H. Low, and K. M. Chandy, "Equivalence of branch flow and bus injection models," in 2012 50th Annual Allerton Conference on Communication, Control, and Computing (Allerton), pp. 1893-1899, Oct 2012.

[20] K. Heuck, K.-D. Dettmann, and D. Schulz, Elektrische Energieversorgung. Wiesbaden: Vieweg+Teubner, 8th ed., 2010.

[21] M. Farivar and S. H. Low, "Branch Flow Model: Relaxations and Convexification - Part I," IEEE Transactions on Power Systems, vol. 28, pp. 2554-2564, Aug 2013

[22] S. H. Low, "Convex relaxation of optimal power flow - part I: Formulation and Equivalence," IEEE Transactions on Control of Network Systems, vol. 1, pp. 177-189, June 2014.

[23] S. H. Low, "Convex relaxation of optimal power flow - part II: Exactness," IEEE Transactions on Control of Network Systems, vol. 1, pp. 177-189, June 2014.

[24] R. L. Boylestad, Introductory Circuit Analysis. Upper Saddle River, NJ, USA: Pearson Prentice Hall Press, 11th ed., 2007.

[25] I. B. Sperstad and M. Korpås, "Energy storage scheduling in distribution systems considering wind and photovoltaic generation uncertainties," Energies, vol. 12, no. 7, 2019.

[26] A. Gleixner, M. Bastubbe, L. Eifler, T. Gally, G. Gamrath, R. L. Gottwald, G. Hendel, C. Hojny, T. Koch, M. E. Lübbecke, S. J. Maher, M. Miltenberger, B. Müller, M. E. Pfetsch, C. Puchert, D. Rehfeldt, F. Schlösser, C. Schubert, F. Serrano, Y. Shinano, J. M. Viernickel, M. Walter, F. Wegscheider, J. T. Witt, and J. Witzig, "The SCIP Optimization Suite 6.0," technical report, Optimization Online, July 2018.

[27] T. Achterberg, "Scip: solving constraint integer programs," Mathematical Programming Computation, vol. 1, pp. 1-41, Jul 2009.

[28] B. Kocuk, S. S. Dey, and X. A. Sun, "Inexactness of sdp relaxation and valid inequalities for optimal power flow," IEEE Transactions on Power Systems, vol. 31, pp. 642-651, Jan 2016.

[29] J. Amme, G. Pleßmann, J. Bühler, L. Hülk, E. Kötter, and P. Schwaegerl, "The eGo grid model: An open-source and open-data based synthetic medium-voltage grid model for distribution power supply systems," Journal of Physics: Conference Series, 2018.

[30] A. Wächter and L. T. Biegler, "On the implementation of an interiorpoint filter line-search algorithm for large-scale nonlinear programming," Mathematical Programming, vol. 106, pp. 25-57, Mar 2006.

[31] HSL Mathematical Software Library, "HSL_MA97 - Bit-compatible parallel sparse symmetric/Hermitian solver using OpenMP," accessed on 23 January 2020.

[32] S. Boyd and L. Vandenberghe, Convex Optimization. Cambridge University Press, 7th ed., 2009 